\title{
Quantum Ferromagnetism and Phase Transitions in Double-Layer Quantum Hall Systems
}

\author{
Kun Yang ${ }^{1}$, K. Moon ${ }^{1}$, L. Zheng ${ }^{1}$, A.H. MacDonald ${ }^{1}$, S.M. Girvin ${ }^{1}$, D. Yoshioka ${ }^{2}$, and Shou-Cheng Zhang ${ }^{3}$ \\ ${ }^{1}$ Department of Physics, Indiana University, Bloomington, IN 47405 \\ ${ }^{2}$ Institute of Physics, College of Arts and Sciences, University of Tokyo, Komaba, Meguroku Tokyo 153, Japan \\ ${ }^{3}$ IBM Alamaden Research Center, San Jose, CA 95120 and Department of Physics, Stanford University, Palo Alto, \\ CA 94305
}

(October 20, 2018)

\begin{abstract}
Double layer quantum Hall systems have interesting properties associated with interlayer correlations. At $\nu=1 / m$ where $m$ is an odd integer they exhibit spontaneous symmetry breaking equivalent to that of spin $1 / 2$ easy-plane ferromagnets, with the layer degree of freedom playing the role of spin. We explore the rich variety of quantum and finite temperature phase transitions in these systems. In particular, we show that a magnetic field oriented parallel to the layers induces a highly collective commensurate-incommensurate phase transition in the magnetic order.
\end{abstract}

75.10.-b, 73.20.Dx, 64.60.Cn

Recent technological progress has allowed production of double-layer quantum Hall systems of extremely high mobility. The separation $d$ of the two 2D electron gases is so small $(d \sim 100 \AA)$ as to be comparable to the spacing between electrons in the same layer and quantum states with strong correlations between the layers have been observed experimentally and discussed theoretically [1] 3]. Wen and Zee have pointed out that at Landau level filling factor $\nu=1 / m$ and in the absence of interlayer tunneling, this system exhibits a spontaneously broken $\mathrm{U}(1)$ gauge symmetry 顿. ( $m$ is an odd integer.) The corresponding Goldstone mode is a neutral density wave in which the densities in the two layers oscillate out of phase. A finite temperature Kosterlitz-Thouless (KT) phase transition is expected to be associated with this broken symmetry.

In this paper we focus for convenience on the case $\nu=1$ and show that this system can be viewed as an easy-plane quantum itinerant ferromagnet. Following Ref. [5] (but with a rotated coordinate system) we will use an 'isospin' magnetic language in which isospin 'up' ('down') refers to an electron in the 'upper' ('lower') layer [6]. Using this language and building upon recent progress in understanding the case of single-layer systems at $\nu=1$ with real spin [7,8] we explore the consequences of the mixing of charge and isospin degrees of freedom and discuss the rich variety of phase transitions controlled by temperature, layer separation, tunneling between layers, layer charge imbalance and magnetic field tilt angles. In addition to the KT transition we find a 'commensurateincommensurate' phase transition as a function of $B_{\|}$, the component of the magnetic field in the plane. Further- more we demonstrate that the Meissner screening of the in-plane component of the magnetic field $\left(B_{\|}\right)$predicted by Ezawa and Iwazaki does not occur. A portion of this rich set of phenomena is captured in the schematic zerotemperature phase diagram illustrated in Fig. 1. The present paper will be devoted to explication of the physical picture underlying this phase diagram. Technical details of the microscopic calculations on which it is based will be presented elsewhere [9].

It is helpful to begin by discussing the limit of zero temperature, zero tunneling amplitude between the layers and layer spacing $d=0$. We work entirely in the lowest Landau level and take the unit of length to be $l \equiv(\hbar c / e B)^{1 / 2}$. Coulomb repulsion induces a strong ex-

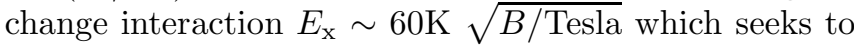
ferromagnetically align all the isospins. Because the kinetic energy has been quenched by the Landau level degeneracy, the exact ground state is simply a single Slater determinant consisting of a filled Landau level with all electrons fully polarized in the same isospin state. Since in this special limit the interactions do not depend on which layer the particles are in, the system exhibits full $S U(2)$ invariance $\left[H, S^{\mu}\right]=0$ and the direction of the polarization is arbitrary. The total isospin for $N$ electrons is $S=N / 2$ and the $2 S+1$ eigenstates of $S^{z} \equiv\left(N_{\uparrow}-N_{\downarrow}\right) / 2$ are completely degenerate.

The exact single-magnon excited states can also be found 10,5]. Taking the magnon vacuum to be the $S^{z}=-N / 2$ ground state, we have

$$
|\mathbf{k}\rangle=\overline{S_{\mathbf{k}}^{+}}|0\rangle
$$

where $S_{\mathbf{k}}^{+}$is the Fourier transform of the isospin raising operator and the overbar indicates projection onto the lowest Landau level. In the single-magnon subspace there is only one state for each wavevector so that $|\mathbf{k}\rangle$ is an exact eigenstate. The eigenvalue $\epsilon_{\mathbf{k}}$ vanishes quadratically at small wavevectors and saturates at $E_{x}$ at large wavevectors. The quadratic dispersion at small $k$ implies an effective isospin stiffness energy for variations in the unit-vector order parameter field $m^{\mu}(\mathbf{r}) \equiv\left(4 \pi \ell^{2}\right)\left\langle S^{\mu}(\mathbf{r})\right\rangle$

$$
T=\frac{1}{2} \rho_{s} \int d^{2} r\left(\nabla m^{\mu}\right) \cdot\left(\nabla m^{\mu}\right)
$$

with $\rho_{s}=e^{2} /(16 \sqrt{2 \pi} \epsilon \ell)$. The microscopic origin of this 
stiffness is the loss of exchange energy when the spin orientation varies with position [8,9].

These properties of the ground state and small wave vector excited states are similar to those of the 2D quantum ferromagnetic Heisenberg model. However, this system is most comparable to itinerant electron ferromagnets and this has some especially interesting consequences [8,9]. In particular at $\nu=1$ charged excitations can only be created by distorting the isospin orientation and, quite remarkably, the fermionic charge density is simply the Pontryagin topological density [8,9]

$$
\delta \rho(\mathbf{r})=\frac{1}{8 \pi} \epsilon^{\mu \nu} \mathbf{m} \cdot\left(\partial_{\mu} \mathbf{m}\right) \times\left(\partial_{\nu} \mathbf{m}\right) .
$$

Thus even though the isospin spectrum is gapless, the isospin stiffness implies a finite charge gap, the lowest energy state carrying charge being a 'skyrmion' spin texture [8,9] whose energy is one-half of the Hartree-Fock charge excitation energy (in the $\mathrm{SU}(2)$ invariant case).

There are several ways in which the $S U(2)$ symmetry can be lifted. The most important consequence of finite layer separation $d$ is the creation of a local capacitive charging energy which for slowly varying charge density $\left(m^{z}\right)$ is [4, [5]

$$
U_{z}=\frac{e^{2} d \eta(d / \ell)}{8 \pi \epsilon \ell^{4}} \int d^{2} r \quad\left[m^{z}(\mathbf{r})\right]^{2}
$$

where $\eta(d / \ell)$ is an exchange correction [5]. This acts as an 'easy plane' anisotropy converting the system from Heisenberg to XY symmetry. For finite $U_{z}, S^{2}$ is no longer a good quantum number. However to a first approximation, the primary effect of $U_{z}$ is simply to lift the degeneracy of the Heisenberg ground states, preferentially selecting states with magnetization lying in the $\mathrm{XY}$ plane in order to minimize the charging energy. The system still has $U(1)$ invariance for rotation about the $\hat{z}$ isospin axis; i.e. the number of electrons in each layer is a good quantum number. The collective mode spectrum is still gapless but the dispersion is now that of a linear Goldstone mode [5, 1, 11]. In the regime of spontaneously broken $U(1)$ symmetry we expect, as discussed below, a finite gap for charged excitations and hence a discontinuity $\Delta \mu$ in chemical potential vs. filling factor which implies a dissipationless quantum Hall state.

If the spacing $d$ exceeds a critical value $d^{*}$ (Fig. 1) the system is unable to support a state with strong interlayer correlations and the spontaneous $U(1)$ symmetry breaking is destroyed by quantum fluctuations [5]. At this same point we expect the quantum Hall effect to be destroyed as the fermionic gap $\Delta \mu$ collapses. In the Hartree-Fock approximation [12] there is a continuous phase transition with a gap collapse occurring at finite wavevector so that the ground state becomes a chargedensity-wave (CDW). Numerical exact diagonalization calculations suggest that there is no CDW state but the transition is continuous [9]. Little else about this transition is firmly established at present. The most naive picture that the transition is in the universality class of the $2+1-\mathrm{D}$ XY model may be incorrect because of the nearby gapless fermionic degrees of freedom and the fact that (see below) vortices carry fermionic charge.

The XY symmetry and finite stiffness in the regime $0<$ $d<d^{*}$ imply a finite temperature KT transition. The local XY spin orientation can be described by a single angle variable $\theta(\mathbf{r})$ and the effective Hamiltonian is

$$
H=\frac{1}{2} \rho_{s} \int d^{2} r|\nabla \theta|^{2}
$$

where, in the Hartree-Fock approximation $\rho_{s}=$ $e^{2} /(16 \pi \epsilon \ell) \int_{0}^{\infty} d x x^{2} \exp \left(-x^{2} / 2-d x / \ell\right)$. The KT critical temperature (within the Villain approximation) is [9] $T_{\mathrm{KT}}=(\pi / 2) \rho_{s} \sim 0.5 \mathrm{~K}$ for typical sample parameters of Murphy et al. [3]. A more detailed estimate will require knowledge of quantum fluctuation corrections to the stiffness and knowledge of the vortex fugacity (core energy) which will be modified by the fact that the vortices carry electron charge $\pm e / 2$. The vortex charge is readily deduced by noting that an electron circling the vortex feels a Berry's phase of $\exp (i \pi)=-1$ induced by the $2 \pi$ rotation of its isospin. Thus it sees the vortex as half a flux quantum which for $\sigma_{x y}=\nu e^{2} / h$ induces charge $\pm \nu / 2= \pm 1 / 2[13]$. One can also see this by noting [9] that each vortex is one of four flavors of 'meron' (half a skyrmion) with \pm vorticity at infinity and a large diameter core (if $d / \ell$ is small) in which the isospins smoothly rotate either up or down out of the XY plane. The defect has half the topological charge and hence half the fermionic charge of a skyrmion.

Normally the KT transition would be visible only in the channel which supports "isospin supercurrents", namely oppositely directed charge currents in each layer. However in the present case a uniform current flow in the same direction in each layer produces a Hall field which will couple to the vortex charge. Thus the KT transition should also manifest itself as a sudden drop in dissipation in this channel for $T \leq T_{\mathrm{KT}}$. In contrast to the usual case in superfluid and superconducting films however, the linear dissipation will not drop to zero (in this channel). This is because gapped non-vortex (i.e., unconfined) fermionic excitations will also couple to the Hall field producing weak thermally activated dissipation.

Another way in which the symmetry can be modified results from the fact that it is possible to unbalance the charge density in the two layers by addition of a bias field $\mathcal{E}$ from a gate. This corresponds to a perturbation $V_{z}=e \mathcal{E} d S^{z}$. If the symmetry has already been lowered from $S U(2)$ to $U(1)$ by the finite $d$ this perturbation causes no dramatic changes. It is expected however to renormalize the stiffness $\rho_{s}$ downwards. The most naive picture of this is simply that when the isospins tilt up in the $\hat{z}$ direction their projection onto the $\mathrm{XY}$ plane is 
reduced. Thus we expect the $\nu=1 \mathrm{XY}$ ordered state to be robust in the presence of variations in $N_{\uparrow}-N_{\downarrow}$. This is in sharp contrast to the situation at other filling factors such as $\nu=1 / 2$ which is described by the Halperin 331 state [14 and which requires equal numbers of electrons in each layer. This characteristic feature of the $\nu=1$ state appears to have been observed [3]. We note that the reduction in $\rho_{s}$ will renormalize $T_{\mathrm{KT}}$ downwards. Near $d^{*}$, charge unbalance could be used to tune to the quantum critical point without having to vary $\left(d-d^{*}\right)$.

The final way that one can modify the isospin symmetry is through a finite tunneling amplitude $t$ which will destroy the XY symmetry by adding a term to the Hamiltonian which selects out a preferred direction and thereby induces an isospin excitation gap

$$
H_{T}=-\int d^{2} r \mathbf{h} \cdot \mathbf{S}(\mathbf{r})
$$

where $\mathbf{h}=2 t \hat{x}$. This perturbation attempts to maximize $\left\langle S^{x}\right\rangle$ since it prefers the particles to be in the symmetric tunneling state. In the presence of tunneling, the system always has a finite value for $\left\langle S^{x}\right\rangle$ no matter how large $d$ is (or even how high the temperature). The fermionic gap is stabilized by this effect so that the critical spacing $d^{*}$ is enhanced [5] in the presence of tunneling (see Fig. 11). However the fermionic gap collapse at the enhanced $d^{*}$ is now no longer directly associated with the complete loss of spontaneous XY magnetic order.

The third axis in Fig. 1 is tilt of the magnetic field achieved by adding a field $B_{\|}$in the plane of the layers. Tilting the field has traditionally been an excellent method of distinguishing effects of (real) spins because orbital motion is primarily sensitive to $B_{\perp}$ while the (real) spin Zeeman splitting is proportional to the full magnitude of $B$. Thus parallel field can destablize spin singlet states in favor of ferromagnetic states. For the case of the double layer $\nu=1$ systems studied by Murphy et al ., [3] the ground state is known to already be an isotropic ferromagnetic state of the true spins and the addition of a parallel field will only serve to further stabilize it. Nevertheless these systems are very sensitive to $B_{\|}$. The activation energy drops rapidly by up to an order-of-magnitude with increasing $B_{\|}$. At $B_{\|}=B_{\|}^{*}$ there appears to be a phase transition to a new state and the activation gap is independent of further increases in $B_{\|}$.

The effect of $B_{\|}$on the isospin system can be visualized in two different pictures. We use a gauge in which $\mathbf{B}_{\|}=\nabla \times \mathbf{A}_{\|}$where $\mathbf{A}_{\|}=B_{\|}(0,0, x)$. In this gauge there is no change in the basis orbitals in each layer but the tunneling matrix element acquires a position-dependent phase $t \rightarrow t e^{i Q x}$ where $Q=2 \pi / L_{\|}$and $L_{\|}=\Phi_{0} / B_{\|} d$ is the length associated with one flux quantum $\Phi_{0}$ between the layers. This modifies the tunneling Hamiltonian to $H_{T}=-\int d^{2} r \mathbf{h}(\mathbf{r}) \cdot \mathbf{S}(\mathbf{r})$ where $\mathbf{h}(\mathbf{r})$ 'tumbles': $\mathbf{h}(\mathbf{r})=2 t(\cos Q x, \sin Q x, 0)$. The effective $\mathrm{XY}$ model now becomes

$$
H=\int d^{2} r\left\{\frac{1}{2} \rho_{s}|\nabla \theta|^{2}-\frac{t}{2 \pi \ell^{2}} \cos [\theta(\mathbf{r})-Q x]\right\},
$$

which is precisely the Pokrovsky-Talapov (P-T) model 115 and has a very rich phase diagram. For small $Q$ and/or small $\rho_{s}$ the phase obeys $\theta(\mathbf{r}) \approx Q x$ but as $B_{\|}$is increased the local field tumbles too rapidly and a continuous phase transition to an incommensurate state with broken translation symmetry occurs. This is because at large $B_{\|}$it costs too much exchange energy to remain commensurate and the system rapidly gives up the tunneling energy in order to return to a uniform state $\nabla \theta \approx 0$ which becomes independent of $B_{\|}$. Using the parameters of the samples of Murphy et al. [3] we find (for $d \ll d^{*}$ within mean-field theory) a critical field for the transition $B_{\|}^{*}=B_{\perp}(2 \ell / \pi d)\left(2 t / \pi \rho_{s}\right)^{1 / 2} \approx 1.6 \mathrm{~T}$ which is within a factor of two of the observed value [3]. Note that the observed value $B_{\|}^{*}=0.8 \mathrm{~T}$ corresponds in these samples to a large value for $L_{\|}: L_{\|} / \ell \sim 20$ indicating that the transition is highly collective in nature. A second strong indication of the collective nature of the excitation gap is the collapse of the observed gap at temperatures much smaller than the gap [3]. We therefore believe that this scenario of a collective commensurate-incommensurate transition explains the phase transition seen in the experiments. Numerical exact-diagonalization calculations on small systems confirm the existence of this phase transition and show that the fermionic excitation gap drops to a much smaller value in the incommensurate phase [9].

The P-T model also undergoes a finite temperature KT phase transition for $B_{\|}>B_{\|}^{*}$ which restores the translation symmetry by means of dislocations in the domain wall structure of the incommensurate phase [15. Thus there are two separate KT transitions in this system, one for $t=0$, the other for $t \neq 0$ and $B_{\|}>B_{\|}^{*}$.

We now discuss the commensurate-incommensurate phase transition from the microscopic point of view. At $d=0$ the $B_{\|}=0$ Landau-gauge many-body ground state wavefunction is a single Slater determinent in which the single-body states are the symmetric linear combination of two single-layer states with the same guiding center. Phase coherence is established by tunneling between single-layer states with the same guiding center. For many purposes this state is still a good approximation to the ground state at finite $d$ since it optimizes the tunneling energy and has good correlation energy; an electron in one layer automatically sees an exchangecorrelation hole in the other layer at the same place. (It would remain the exact ground state in the absence of interactions.) From a microscopic point of view it is the good interlayer correlations of states with phase coherence which leads to the broken symmetry in the absence of tunneling. In the Landau gauge, a parallel field causes 
tunneling to couple states in the two layers whose momenta differ by $Q$ and whose guiding centers therefore differ by $\ell^{2} Q$. Thus, for non-interacting electrons the exact ground state in a parallel field is one in which the exchange-correlation hole is not directly opposite its electron but rather shifts away by $\ell^{2} Q \hat{x}$ as the $\mathbf{B}$ field tilts (so that a field line passing through the electron also passes through the hole). This state maintains all of its tunneling energy but rapidly loses correlation energy as the field tilts. At large tilt it is better to give up on the tunneling by shifting the two layers relative to each other to put the correlation hole back next to its electron. This shift can be shown to be the change from commensurate to incommensurate [9] states disussed above.

Ezawa and Iwazaki [16] have recently proposed that there is a Meissner effect which can screen out the applied $B_{\|}$within a finite 'Josephson screening length'. We find however that this is incorrect. Consider the analog of eq.( (7) for a spatially varying $B_{\|}(x)$

$$
H=\int d^{2} r\left\{\frac{1}{2} \rho_{s}|\nabla \theta|^{2}-\frac{t}{2 \pi \ell^{2}} \cos [\theta(\mathbf{r})-A(x)]\right\}
$$

where $\partial_{x} A(x)=\left(2 \pi d / \Phi_{0}\right) B_{\|}(x)$. Taking a weak slowly varying field $A(x)=A_{q} \cos q x$ and making the harmonic approximation, one readily finds that the variationally optimal $\theta(x)$ gives an energy density

$$
\mathcal{E}\left(A_{q}\right)=\frac{1}{2} \rho_{s} q^{2}\left|A_{q}\right|^{2}=\frac{1}{2} \rho_{s}\left(\frac{2 \pi d}{\Phi_{o}}\right)^{2} B_{\|}^{2}
$$

indicating that the system is an ordinary diamagnet with vanishing Meissner kernel. The physical reason for this is simply that it is very inexpensive at long wavelengths for the phase to twist. Thus in the 'commensurate phase' the gauge invariant phase difference across the junction (and hence the screening currents) nearly vanish.

The authors are grateful to S. Q. Murphy, J. P. Eisenstein, and G. S. Boebinger for showing us their data prior to publication, to S. Sondhi and S. Renn for stimulating discussions, and to J. Carini and Mats Wallin for graphics assistance. This work was supported by NSF DMR9113911 and the Aspen Center for Physics.

[1] Y.W. Suen et al., Phys. Rev. Lett. 68, 1379 (1992); J.P. Eisenstein et al., Phys. Rev. Lett. 68, 1383 (1992).

[2] B. I. Halperin, Helv. Phys. Acta 56, 75 (1983); A.H. MacDonald, Surf. Sci. 229, 1 (1990); Z. F. Ezawa and A. Iwazaki, Phys. Rev. B 47, 7295 (1993); Z. F. Ezawa et al., preprint. Song He et al., Phys. Rev. B 47, 4394 (1993); T. Chakraborty and P. Pietiläinen, Phys. Rev. Lett. 59,
2784 (1987); E.H. Rezayi and F.D.M. Haldane, Bull. Am. Phys. Soc. 32, 892 (1987).

[3] S.Q. Murphy, J. P. Eisenstein, and G. S. Boebinger, L. N. Pfeiffer and K. W. West, to be published and private communication.

[4] X.G. Wen and A. Zee, Phys. Rev. Lett. 69, 1811 (1992); X.G. Wen and A. Zee, Phys. Rev. B 47, 2265 (1993).

[5] A.H. MacDonald, P.M. Platzman, and G.S. Boebinger, Phys. Rev. Lett. 65, 775 (1990). See also L. Brey, ibid. 65, 903 (1990) and H. Fertig, Phys. Rev. B 40, 1087 (1989).

[6] Throughout this discussion we will ignore true spin and assume that the real electron spins are fully polarized by the magnetic field. This simplifying assumption is not necessarily valid at low fields.

[7] D.-H. Lee and C.L. Kane, Phys. Rev. Lett. 64, 1313 (1990).

[8] S.L. Sondhi et al., Phys. Rev. B 47, 16419 (1993).

[9] Kun Yang, K. Moon, L. Zheng, A.H. MacDonald, S. M. Girvin, S.-C. Zhang, and D.Yoshioka, in preparation.

[10] C. Kallin and B. Halperin, Phys. Rev. B 31, 3635 (1985); M. Rasolt and A.H. MacDonald, ibid. 34, 5530 (1986).

[11] A.H. MacDonald and Shou-Cheng Zhang, submitted to Phys. Rev. Lett. (1993).

[12] R. Côté, L. Brey, and A.H. MacDonald, Phys. Rev. B 46, 10239 (1992); X.M. Chen and J.J. Quinn, Phys. Rev. B 45, 11054 (1992).

[13] Kun Yang, L.K. Warman, and S.M. Girvin, Phys. Rev. Lett. 70, 2641 (1993).

[14] D. Yoshioka, A.H. MacDonald, and S.M. Girvin, Phys. Rev. B 39, 1932 (1989).

[15] Per Bak, Rep. Prog. Phys. 45, 587 (1982); Marcel den Nijs in Phase Transitions and Critical Phenomena 12, ed. by C. Domb and J.L. Lebowitz (Academic Press, New York, 1988) pp. 219-333.

[16] Z. Ezawa and A. Iwazaki, Phy. Rev. Lett. 70, 3119 (1993).

FIG. 1. Schematic zero-temperature phase diagram (with $d / \ell$ increasing downwards). The lower surface is $d^{*}$ below which $d>d^{*}$ and the interlayer correlations are too weak to support a fermionic gap, $\Delta \mu$. The upper surface gives $B_{\|}^{*}$, the commensurate-incommensurate phase boundary. As $d$ approaches $d^{*}$, quantum fluctuations soften the spin stiffness and therefore increase $B_{\|}^{*}$. 